\title{
Multi-catheter interstitial accelerated partial breast irradiation - tips and tricks for a good insertion
}

\author{
Johann I. Tang, MBBS, FRANZCR, FAMS, Poh Wee Tan, Vicky Yanling Koh, MBBS, FRANZCR, FAMS, Shaik Ahmad \\ Buhari, MBBS, FRCS (Edin) \\ Department of Radiation Oncology, National University Cancer Institute, Singapore
}

\begin{abstract}
Adjuvant radiotherapy is recommended post breast conserving surgery. Accelerated Partial Breast Irradiation (APBI) offers a more attractive shorter course of treatment over 5 days compared to standard conventional external beam radiotherapy, which is often protracted. Multi-catheter interstitial APBI offers excellent dosimetric coverage. This article describes two insertion techniques for multi-catheter interstitial APBI, the operator dependent freehand technique, and the easier to learn template technique. The indications, benefits, and drawbacks of these two techniques
\end{abstract} are discussed.

J Contemp Brachytherapy 2014; 6, 1: 85-90 DOI: $10.5114 /$ jcb. 2014.40767

Key words: breast, multi-catheter, brachytherapy.

\section{Purpose}

In women with early stage breast cancer post breast conserving surgery, adjuvant radiotherapy remains the standard of care resulting in local control, as well as overall survival benefit [1-3]. The use of mammographic screening has enabled earlier identification of women with early stage breast cancer, therefore increasing the breast conservation rates in this group of women [4]. However, the use of conventional fractionation radiotherapy given over 5 to 6 weeks may pose a barrier to patients receiving adjuvant radiotherapy, leading to some women opting for total mastectomy instead [5]. To avoid unnecessary total mastectomies for this group of patients with early stage breast cancer, accelerated partial breast irradiation (APBI) presents a viable alternative radiotherapy treatment regimen applied in overall shorter period of time. This would reduce the logistic barriers, especially for women who are economically active and find it difficult to take time off work, or for women who need to travel long distances for their radiotherapy treatment. APBI therefore makes breast conservation treatment a viable alternative in these women who would otherwise have opted for total mastectomies instead.

There are many different APBI modalities, i.e. intraoperative, mammosite, multi-catheter, and external beam [6-8]. This article focuses on the use of multi-catheter interstitial APBI, its clinical indications, the various catheter insertion techniques, and tips and tricks to perform a good catheter insertion.

Multi-catheter interstitial APBI is the oldest method used. Whilst previous studies using multi-catheter inter- stitial APBI did not achieve good results mainly due to poor patient selection, recent studies have demonstrated that with careful patient selection, excellent clinical results with good local control can be achieved with this technique [9-11]. These current excellent results form the basis of the American Society for Therapeutic Radiation Oncology (ASTRO) [10], American Brachytherapy Society [11] and Groupe Européen de Curiethérapie - European Society for Therapeutic Radiation Oncology (GEC-ESTRO) [12] consensus guidelines that were published indicating which patients are suitable, which patients should be considered cautionary, and which patients are unsuitable for APBI (Table 1). In general, the suitable and cautionary patients in the ASTRO/ESTRO consensus statement can be offered multi-catheter interstitial APBI. In the author's experience, these 2 groups of patients make up about $80 \%$ of the cases seen at the author's centre.

\section{Technique}

\section{Target localization}

Ultrasound guided localization of the target is essential for a successful implant. Under ultrasound guidance, about $0.5-1 \mathrm{cc}$ of Omnipaque ${ }^{\mathrm{TM}}$ is injected into the tumor cavity. This is to allow the tumor cavity to be easily identifiable on the pre-procedure CT scan (Fig. 1). In addition, instead of using a skin surface marker pen, the outline of the cavity is marked by imprinting a straw on the breast skin surface as the marker pen ink washes off easily when the area is cleaned with chlorhexidine solution before the procedure. By imprinting the straw marks on the skin, the 
Table 1. Patient suitability criteria for APBI as per the various consensus guidelines

\begin{tabular}{|c|c|c|c|}
\hline Criteria & $\mathrm{ABS}$ & $\mathrm{ESTRO}^{+}$ & $\mathrm{ASTRO}^{+}$ \\
\hline Age & $\geq 50$ years old & $>40$ years old & $\geq 50$ years old \\
\hline Size & $\leq 3 \mathrm{~cm}$ & $\leq 3 \mathrm{~cm}$ & $\leq 3 \mathrm{~cm}$ \\
\hline Histology & $\begin{array}{c}\text { all invasive subtypes and } \\
\text { DCIS* }\end{array}$ & $\begin{array}{c}\text { all invasive subtypes and } \\
\qquad \text { DCIS }^{*}\end{array}$ & $\begin{array}{l}\text { all invasive subtypes and } \\
\text { DCIS* }\end{array}$ \\
\hline Estrogen receptor & positive/negative & positive/negative & positive/negative \\
\hline Surgical margins & negative & negative/close (<2 mm) & negative/close (< $2 \mathrm{~mm})$ \\
\hline Lymphovascular space invasion & negative & negative & negative/focal \\
\hline Nodal status & negative & < 4 lymph nodes involved & negative \\
\hline
\end{tabular}

${ }^{*}$ Ductal carcinoma in-situ; ${ }^{+}$Excluding unsuitable category

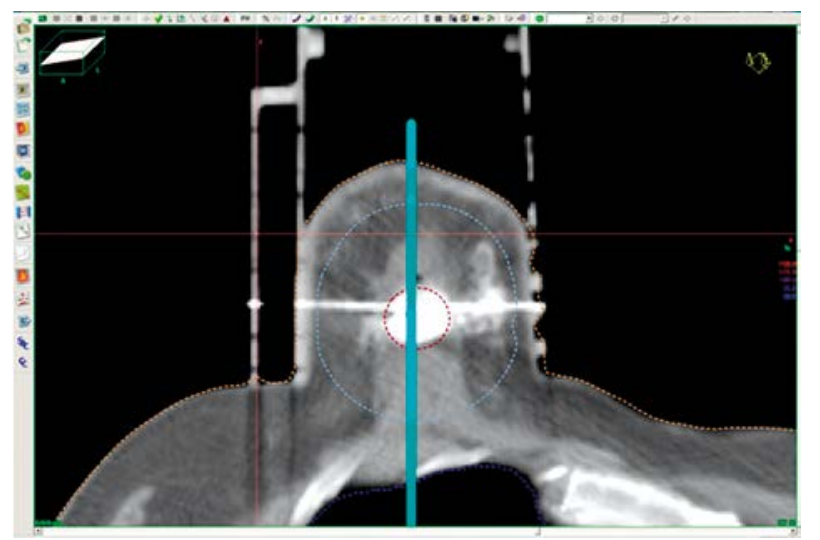

Fig. 1. Cavity identification

impression of the cavity outline remains even after the cleaning of the ipsilateral breast pre-procedure.

A pre-procedure CT scan is then acquired to gain information on the location of the tumor in respect to the nipple and the depth of the normal breast tissue, so as to avoid the risk of a pneumothorax caused by puncturing the underlying ipsilateral lung.

In pre-procedure, it is advisable to mark the outline of the medial borders of the brassiere, as well as the dress line against the breast (Fig. 2). This is to ensure that no catheters are placed medially to this line to avoid them being seen when the patient wears a low cut dress. This ensures that patients are more confident to continue their activities of daily living, and have the freedom of what to wear whilst continuing treatment.

\section{Anesthesia}

Moderate sedation is usually the mode of anesthesia. A cocktail of Oxycodone $5 \mathrm{mg}$, Valium $5 \mathrm{mg}$ and Synflex $550 \mathrm{mg}$ is given to the patient 45 minutes before the commencement of the procedure. Tumescent anesthesia made up of $20 \mathrm{mls}$ of $2 \%$ lignocaine, $1: 100,000$ adrenaline, and $2.5 \mathrm{mls}$ of $8.4 \%$ sodium bicarbonate mixed in $250 \mathrm{mls}$ of normal saline is used as a breast local anesthetic agent. Other equipment includes a pair of $20 \mathrm{ml}$ syringes with an $11 \mathrm{G}$ spinal needle attached for the delivery of the tumescent anesthesia.

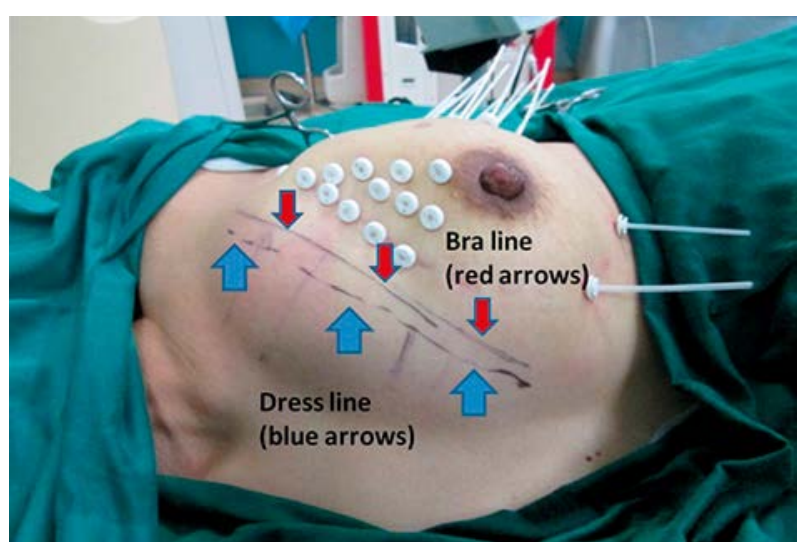

Fig. 2. The marked bra and dress outlines. None of the catheters should be placed superior to the dress line to ensure a good cosmetic outcome and patient satisfaction

\section{Catheter insertion techniques}

There are two methods of catheter insertion: the template approach or the free hand technique.

\section{Template approach}

The template approach with the Kuske template $(\mathrm{Nu}-$ cletron, an Elekta company, Elekta AB, Stockholm, Sweden) is most suitable for patients with a B to D cup size breast. Once the cavity is localized, the ipsilateral breast area is surgically prepped under sterile conditions. To avoid injury to the underlying chest wall structures or causing a pneumothorax, the overlying breast is pinched and gently lifted off the chest wall before applying the template and securing it. Also to note is that the $C 12$ grid position should ideally be in the centre of the tumor cavity to ensure that adequate grid space is available either side of the template for catheter insertion. Usually 3-5 anchoring needles are then placed in an asymmetric pattern, usually involving C 12 (Fig. 3). The purpose of the asymmetric pattern is to aid easy orientation of the template in reference to the patient's breast anatomy, as well as to secure and prevent slipping of the template from the breast. A CT scan is then obtained with the anchoring catheters in-situ. These images are then reconstruct- 


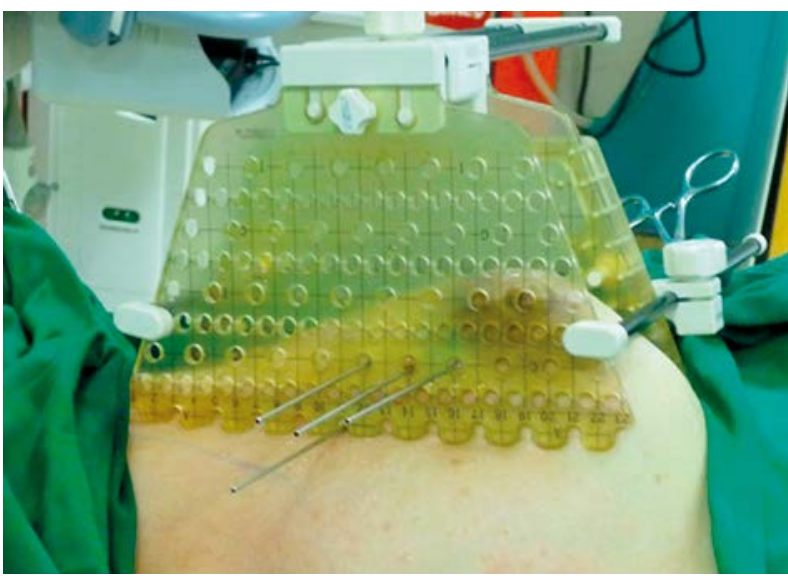

Fig. 3. Anchoring Catheters showing the asymmetric placement of the catheters

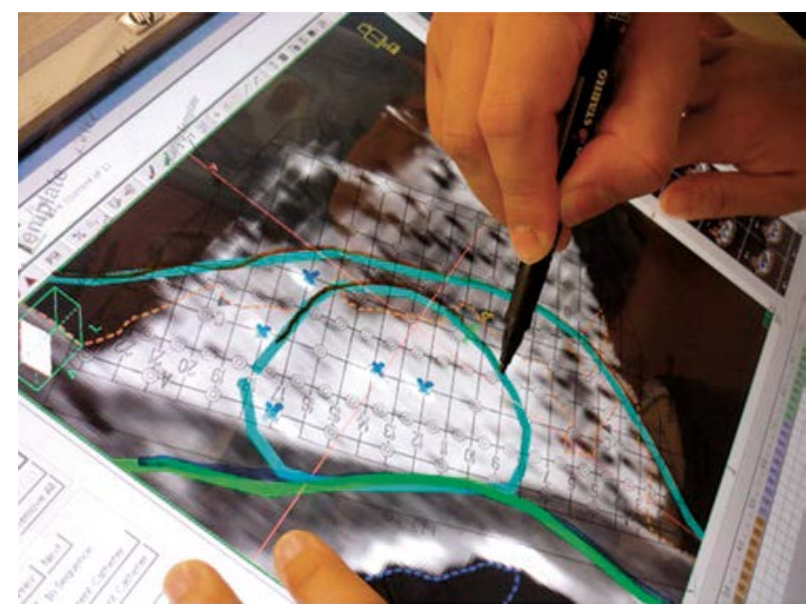

Fig. 5. Overlaying and tracing the various contours onto the template

ed on the Oncentra ${ }^{\circledR}$ Brachy planning system (Nucletron, an Elekta company, Elekta AB, Stockholm, Sweden). The Planning Target Volume (PTV) is formed by expanding 15 to $20 \mathrm{~mm}$ from the Clinical Target Volume (CTV), which is determined from the contrast enhanced tumor cavity and any surrounding surgical clips. The PTV-Eval is then formed by excluding the $5 \mathrm{~mm}$ skin margin, as well as the underlying chest wall muscle layer. The skin is also contoured on the sagittal slice depicting the largest breast contour (usually the slice showing the nipple), and the most anterior chest wall surface is also contoured. Remember to scroll through the images, as some other slices may depict a more anterior skin or chest wall contour. If that happens, the skin or chest wall contour should be altered to include this new more anterior contour. The images are then reoriented in Oncentra to depict the "template view" (Fig. 4). This template view is crucial to determine where the rest of the catheters should be placed. Next, an overlying photocopy of the template on a transparency is placed on the top of the screen, and the "template view" template magnification is matched $1: 1$

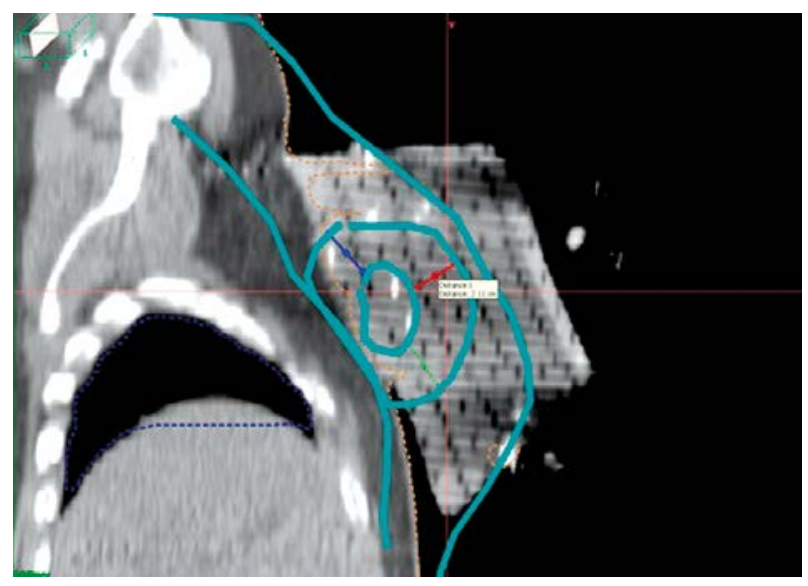

Fig. 4. Template view showing the CTV, PTV as well as skin and chest wall contours. The volume between these 2 contours represents the "safe zone" for catheter insertion

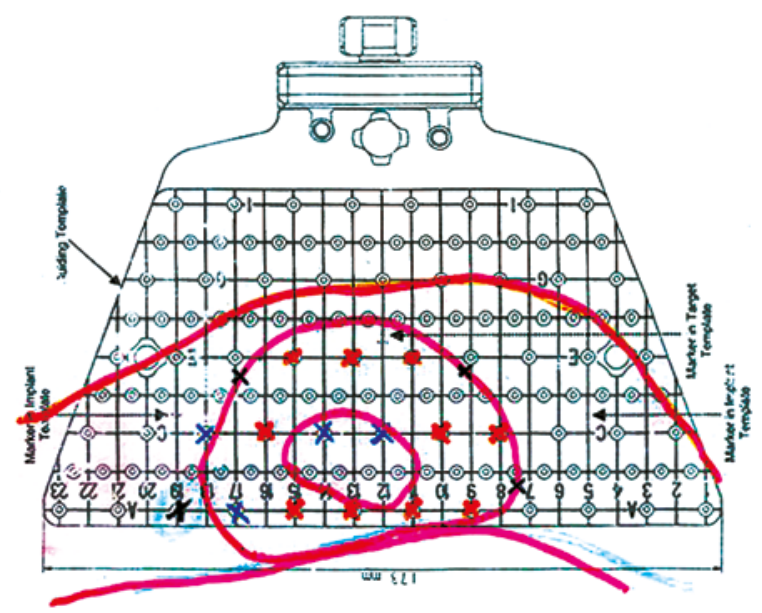

Fig. 6. Picture showing the transparency template with the positions of the catheter placement

to the overlaid template transparency (Fig. 5). A good reference of a good 1:1 magnification are matching the 3 largest holes for securing the screws on the template. The corresponding anchoring needle positions are then marked on the overlaid template transparency, the chest wall contour, PTV-Eval contour, and the chest wall contour outlined onto the transparency. Once done, the rest of the catheter placement can be easily determined (Figs. 6 and 7). In general, any catheters within the PTV-Eval need to be inserted. For the catheters out of the PTV-Eval, if they are within $1 \mathrm{~cm}$ of the PTV-Eval contour, they would need to be inserted as well. By using this method, one can easily determine the exact number of catheters required to cover the PTV-Eval volume, therefore avoiding under or over insertion of catheters. Another point to note is to avoid placing catheters outside of the patient's favourite low cut dress neckline, so that the catheters cannot be seen when the low cut dress is worn (Fig. 8). If the catheters protrude out into the neckline area, the best thing is to manually relocate the entry point of the catheter below the neckline without compromising the dosimetric coverage. 


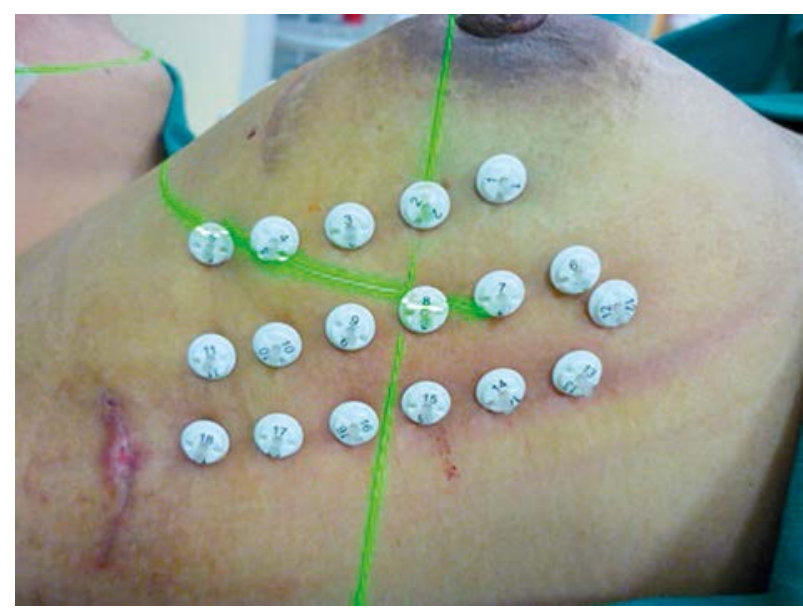

Fig. 7. The final catheter placement and cosmetic result

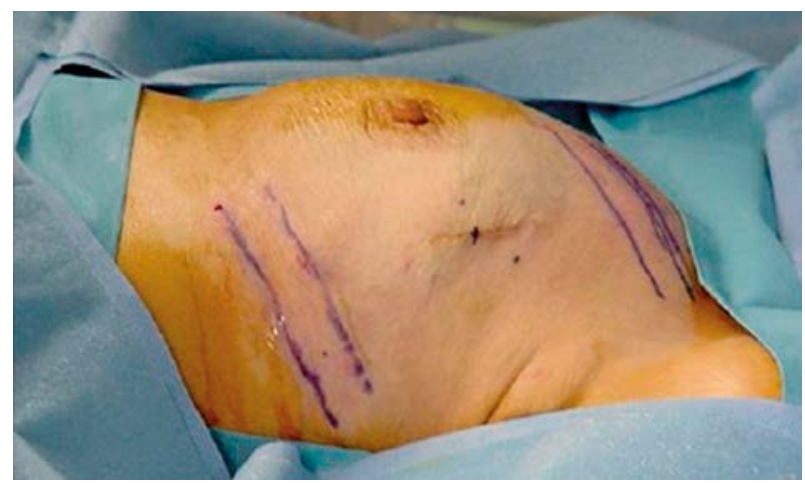

Fig. 9. Two sets of parallel lines show the insertion and exit planes of the catheters

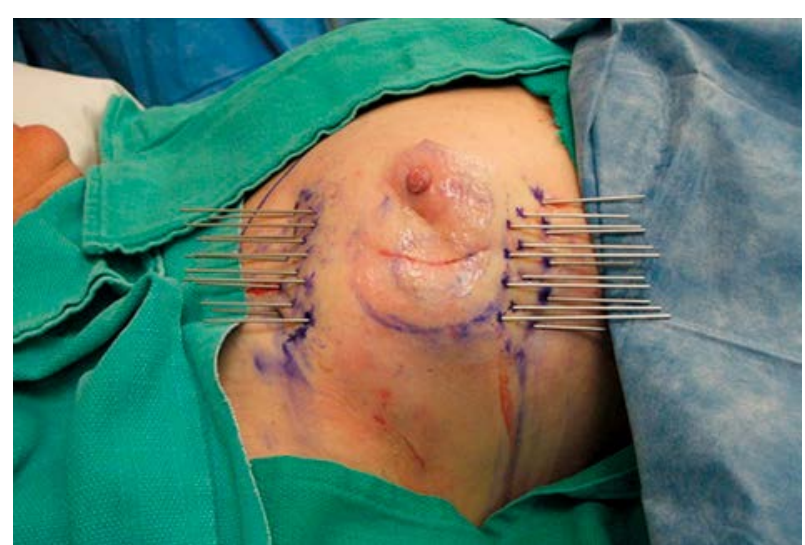

Fig. 11. The final catheter placement and cosmetic result

\section{Free hand approach}

The freehand approach is used in patients with a small breast size or where the tumor cavity location is unsuitable for the application of the Kuske Template, for instance in extremely medial or lateral tumour locations. This approach is operator dependent and requires the use of ultra-

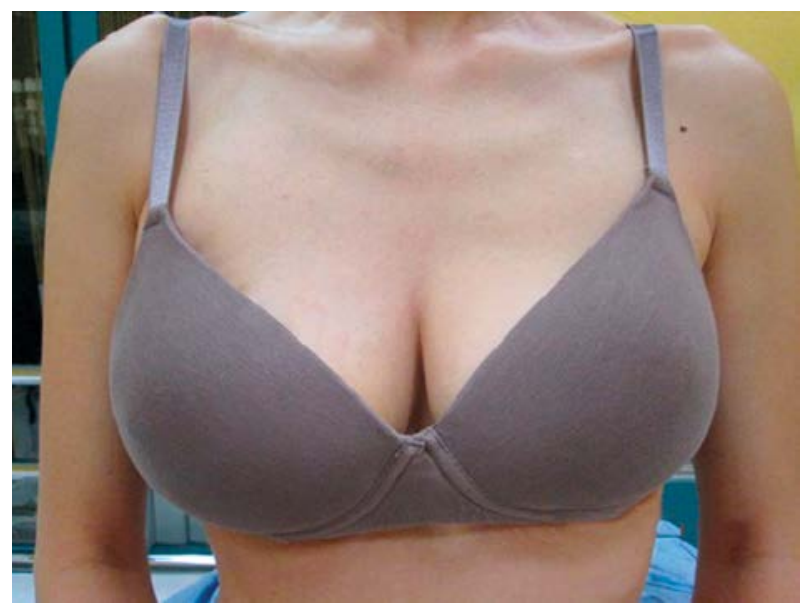

Fig. 8. By placing catheters below the marked bra line, catheters are not seen when the bra is worn, giving excellent cosmesis and patient satisfaction

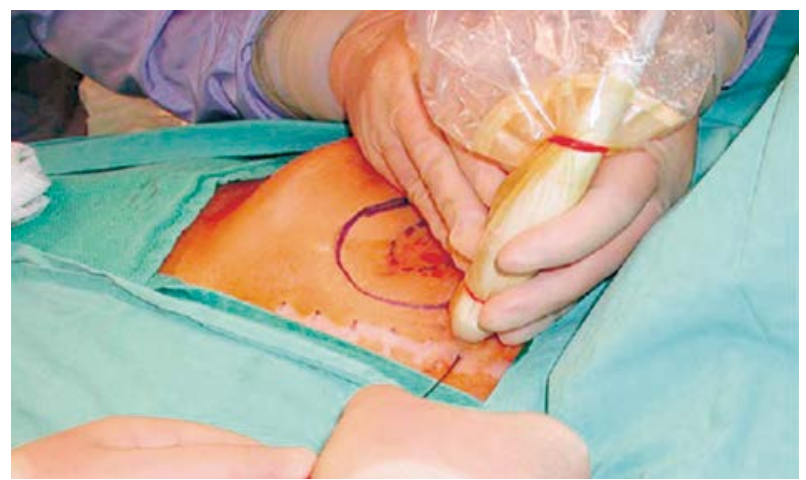

Fig. 10. Catheters are placed under ultrasound guidance

sound to guide the insertion of the catheters. This approach should only be used by physicians who already have some experience with multi-catheter interstitial APBI.

Once the ipsilateral breast is sterile, the tumor cavity outline or CTV is marked on the skin surface. The PTV is then created by expanding 15 to $20 \mathrm{~mm}$ from the CTV. 2-3 parallel lines about $15 \mathrm{~mm}$ apart are drawn about $2 \mathrm{~cm}$ from the PTV on either side (Fig. 9). Following the Paris system, intervals of $15 \mathrm{~mm}$ are then marked on the parallel lines in such a pattern that these marked points form an equilateral triangle. Under ultrasound guidance, the catheters are then guided through the breast where the insertion point matches the corresponding opposite exit point on the other side of the PTV (Figs. 10 and 11). The main dangers are the risk of catheter crossing, and the higher risk of underlying chest wall damage or pneumothorax. In addition, as it is very difficult to determine the relationship of the needles to the surgical cavity, one tends to err on the side of caution by placing in more needles to compensate. Even with more needles placed, one sometimes finds that obtaining good dosimetric coverage may be difficult even in experienced hands. In the author's experience, this approach should not be recommended for anyone who is just commencing to perform a multi-catheter interstitial APBI due to the above reasons. 
Table 2. Comparison of our results with other institutions

\begin{tabular}{ccccc} 
& $\begin{array}{c}\text { Freehand } \\
\text { (non-template) }\end{array}$ & $\begin{array}{c}\text { University of Wiscon- } \\
\text { sin }(\mathrm{n}=50)\end{array}$ & Template & Tufts University $(\mathrm{n}=75)$ \\
\hline $\mathrm{D}_{90}$ & $3.13 \mathrm{~Gy}(92 \%)$ & $96 \%$ & $3.30 \mathrm{~Gy}(97 \%)$ & $95 \%$ \\
\hline $\mathrm{DHI}$ & $74 \%$ & $70 \%$ & $76 \%$ & $80 \%$ \\
\hline $\mathrm{V}_{200}$ & $9.6 \mathrm{ccm}^{3}$ & $10 \mathrm{ccm}^{3^{*}}$ & $8.0 \mathrm{ccm}^{3}$ & $12 \mathrm{ccm}^{3}$ \\
\hline $\mathrm{MSD}$ & $100 \%$ & $100 \%$ & $90 \%$ & $100 \%$ \\
\hline $\mathrm{MCD}$ & $80 \%$ & $80 \%$ & $60 \%$ & $80 \%$
\end{tabular}

*Personal communication with Dr Patel

\section{Planning}

The patient receives a total dose of $34 \mathrm{~Gy}$ in 10 fractions, two fractions per day, 3.4 Gy per fraction, separated by at least 6 hours, given on 5 treatment days. Target coverage is $\geq 90 \%$ of the prescribed dose, covering $\geq 90 \%$ of the PTV-Eval $\left(D_{90} \geq 90 \%\right)$. The skin dose should not exceed prescription dose [13]. To assure appropriate dose homogeneity throughout the implant, two parameters will be used: the volume of tissue receiving higher doses, and a Dose Homogeneity Index (DHI). The actual volume of tissue receiving $150 \%\left(\mathrm{~V}_{150}\right)$ and $200 \%$ $\left(\mathrm{V}_{200}\right)$ of the prescribed dose will be limited to $\leq 70 \mathrm{cc}$ and $\leq 20 \mathrm{cc}$, respectively. The DHI, as represented by the volume ratio $\left(1-\mathrm{V}_{150} / \mathrm{V}_{100}\right)$, will be $\geq 0.75\left(\mathrm{~V}_{150}\right.$ will be the volume of tissue receiving $150 \%$ of the prescribed dose, and $V_{100}$ will be the volume of tissue receiving the prescribed dose). In addition, $<60 \%$ of the ipsilateral whole breast reference volume should receive $\geq 50 \%$ of the prescribed dose. The whole breast reference volume can be found in the online Breast Atlas from the Radiation Therapy Oncology Group (RTOG) website [14].

\section{Discussion}

The main advantage of the template approached is that the dosimetric coverage of the PTV-Eval is excellent and easily achievable with minimal hotspot problems, performed even by novice hands. Target coverage of $D_{90}$ $\geq 90 \%$ is readily achievable. $\mathrm{DHI}$ of more than 0.75 is also easily achievable with this template approach. Other advantages include the lower risk of underlying chest wall damage including a lower pneumothorax risk, as well as the avoidance of over or under insertion of catheters, as the exact number of catheters can be easily determined from the overlaid transparency of the template without compromising any PTV-Eval coverage. Avoiding too many catheters being inserted also reduces infection and bleeding risk. The usual time (from start to end) for the template approach is about 90 minutes, compared to about 150 minutes for the freehand approach.

Additional advantages of the template approach are that it has a less steep learning curve, can be easily mastered, and can be demonstrated to residents without difficulty.

The freehand approach on the other hand is mainly suitable for all breast sizes, as the Kuske template ap- proach may be less suitable for A cup sized breasts, and tumors which are located most medially and laterally. The main drawbacks are that it has a very steep learning curve, longer procedural time, and requires excellent hand-eye coordination during the ultrasound guided catheter insertion. Damage to the underlying chest wall and acute pneumothorax risk is higher with the freehand approach, especially for tumor cavities located adjacent to the underlying chest wall structure. One would usually resort to free hand only for reasons of unsuitable breast size or unfavourable tumor cavity location. Other drawbacks include underestimation of the number of catheters required to cover the PTV-Eval leading to poor dosimetric coverage, increased hotspots leading to lower DHI index, and higher risk of fat necrosis and local control failure rates, as well as crossing of catheters, leading to difficulties and errors in reconstructing the catheters on the Oncentra treatment planning system.

Our institution did a phase II study, which compared the two methods [15]. Compared to the freehand approach, the template approach offered significant shorter insertion and planning times with significantly improved dosimetric PTV-Eval coverage without significantly compromising organs at risk dosimetrically. When we compared the results of our freehand technique and our template technique with the results from the University of Wisconsin [16] and Tufts University [17], respectively, our results were comparable to these institutions (Table 2).

\section{Conclusions}

In conclusion, the template approach offers fewer uncertainties with regards to dosimetric planning with superior dosimetric outcomes, better DHI values, and a shorter procedural time compared to the freehand approach. Unless the patient's breast size is unsuitable or the tumour location is unfavourable, the template approach should be the standard for catheter insertion in multi-catheter interstitial APBI.

\section{References}

1. Early Breast Cancer Trialists' Collaborative Group (EBCTCG). Effect of radiotherapy after breast-conserving surgery on 10year recurrence and 15-year breast cancer death: meta-analysis of individual patient data for 10,801 women in 17 randomised trials. Lancet 2011; 378: 1707-1716. 
2. Recht A, Solin LJ. Breast-conserving surgery and radiotherapy in early-stage breast cancer: the importance of local control. Semin Radiat Oncol 2011; 21: 3-9.

3. Vinh-Hung V, Verschraegen C. Breast-conserving surgery with or without radiotherapy: pooled-analysis for risks of ipsilateral breast tumor recurrence and mortality. J Natl Cancer Inst 2004; 96: 115-121.

4. Olsen O, Gøtzsche PC. Screening for breast cancer with mammography. Cochrane Database Syst Rev 2001; (4): CD001877.

5. Boscoe FP, Johnson CJ, Henry KA et al. Geographic proximity to treatment for early stage breast cancer and likelihood of mastectomy. Breast 2011; 20: 324-328.

6. Vicini FA, Arthur DW. Breast brachytherapy: North American experience. Semin Radiat Oncol 2005; 15: 108-115.

7. Polgár C, Major T. Current status and perspectives of brachytherapy for breast cancer. Int J Clin Oncol 2009; 14: 7-24.

8. Skowronek J, Wawrzyniak-Hojczyk M, Ambrochowicz K. Brachytherapy in accelerated partial breast irradiation (APBI) - review of treatment methods. J Contemp Brachytherapy 2012; 4: 152-164.

9. Gifford K, Nelson C, Kirsner S et al. On the feasibility of treating to a $1.5 \mathrm{~cm}$ PTV with a commercial single-entry hybrid applicator in APBI breast brachytherapy. J Contemp Brachytherapy 2012; 4: 29-33.

10. Wazer D, Arthur D, Vicini F. Accelerated Partial Breast Irradiation. $2^{\text {nd }}$ ed. Springer Verlag, Berlin 2009; pp. 207-345.

11. Polgar C, Fodor J, Major T et al. Breast conserving therapy with partial whole breast irradiation. Ten-year results of the Budapest randomized trial. Radiother Oncol 2013; 108: 197-202.

12. Smith BD, Arthur DW, Buchholz TA et al. Accelerated partial breast irradiation consensus statement from the American Society for Radiation Oncology (ASTRO). Int J Radiat Oncol Biol Phys 2009; 74: 987-1001.

13. Shah C, Vicini F, Wazer DE et al. The American Brachytherapy Society consensus statement for accelerated partial breast irradiation. Brachytherapy 2013; 12: 267-277.

14. Polgar C, Van Limbergen E, Potter R et al. Patient selection for accelerated partial-breast irradiation (APBI) after breast conserving surgery: Recommendations of the Groupe Europeen de Curietherapie-European Society for Therapeutic Radiology and Oncology (GEC-ESTRO) breast cancer working group based on clinical evidence (2009). Radiother Oncol 2010; 94: 264-273.

15. NSABP B-39: A Randomized Phase III Study of Conventional Whole Breast Irradiation (WBI) versus Partial Breast Irradiation (PBI) for Women with Stage 0, I, or II Breast Cancer. http://www.rtog.org/ClinicalTrials/ProtocolTable/Study Details.aspx?study $=0413$. Last accessed on $10^{\text {th }}$ Nov 2013.

16. http:// www.rtog.org/CoreLab/ContouringAtlases/BreastCancerAtlas.aspx. Last accessed on $10^{\text {th }}$ Nov 2013.

17. Koh V, Buhari S, Tan P et al. Comparing a volume based template approach and ultrasound guided freehand approach in multicatheter interstitial accelerated partial breast irradiation. J Contemp Brachytherapy 2014. In press. 